\title{
Principles and Implementation of an Ultrafast Transmission Electron Microscope
}

\author{
Luca Piazza $^{1}$, Mathieu Cottet $^{1}$,Daniel J. Masiel ${ }^{2}$, Thomas LaGrange ${ }^{3}$, Fabrizio Carbone ${ }^{1}$ \\ 1. Laboratory for Ultrafast Microscopy and Electron Scattering, EPDL, Lausanne, Switzerland \\ 2. Intergrated Dynamic Electron Solutions, Inc. Belmont CA \\ 3. Condensed Matter and Materials Division, Lawrence Livermore National Laboratory, Livermore \\ CA
}

In this communication we report on the performance of a fs-resolved transmission electron microscope, installed at the École Polytechnique Fédérale de Lausanne (EPFL), in the Laboratory for Ultrafast Microscopy and Electron Scattering (LUMES). The microscope, constructed by Integrated Dynamic Electron Solutions (IDES), is a variant of the Dynamic Transmission Electron Microscope developed at Lawrence Livermore National Laboratory capable of probing photoinitiated processes in materials on femtosecond timescales with2 Ångström resolution.

Ultrafast temporal resolution is achieved by generating a photoelectron probe using $266 \mathrm{~nm}$ laser pulses that are optically delayed relative to infrared pump pulses hitting the sample. To meet the energy, duration and repetition rate requirements we use a prototype laser system from KMLabs that delivers $80 \mathrm{fs}$ pulses a ta tunable repetition rate, $200 \mathrm{kHz}$ to $2 \mathrm{MHz}$, with an energy of $1.55 \mathrm{eV}$ per photon and an average power of $3 \mathrm{~W}$.

The microscope is a modified JEOL JEM-2100TEM equipped with IDES constructed laser port and $\mathrm{C} 0$ lens sections that enable two pulsed laser beams to enter the column; an ultraviolet beam to illuminate the LaB6 cathode, generating electron pulses, and an infrared beam to stimulate excitations in the sample figure 1). In stroboscopic operation, to avoid space-charge effects and achieve femtosecond time resolution, every bunch of electrons should contain as few as 1 electron. This poses limitations in terms of integration time however this is overcome by the high repetition rate of the laser system and the improved coupling into the condenser system provided by the $\mathrm{C} 0$ lens. It is of capital importance to control the amount and spatial distribution of the charge photoemitted from the LaB6 tip. To do this, the optical set-up is designed in order to match the UV spot size to the flat surface of the cathode $(50 \mu \mathrm{m})$; this limits the emission area of the tip, without the relying on high Wehnelt bias settings. The emitted electrons are then coupled in the column via an additional electromagnetic lens (C0lens) located just below the electron gun. This solution allows optimal coupling of the photoemitted electrons to the condenser system.

High resolution images on a gold nanoparticle test sample have been collected to demonstrate that the modifications to the electron-optical system did not limit the spatial resolution of the microscope (figure 2).

The energy spread of the electron beam has been also characterized via the post column Gatan imaging filter. We notice here that the addition of the $\mathrm{C} 0$ lens allows us to efficiently couple electrons emitted from the filament and conserve the source brightness. Because the $\mathrm{C} 0$ lens allows much higher throughput, all the electrons emitted from the filament can be coupled to the standard TEM optics, producing currents as high as few microamps on the sample and detector. Thus lower filament heating currents can be used while maintaining reasonable signals on the detector, reducing the energy spread of the source and increasing spectroscopic energy resolution.

Figure 3 shows the width of the zero loss peak as measured using the GIF Quantum EELS spectrometer for different filament heating currents, Wehnelt bias voltage settings, and $\mathrm{C} 0$ lens voltage. As is expected, using a lower filament heating current results in a narrower zero-loss peak. In addition, since the $\mathrm{C} 0$ lens improves coupling and allows high throughput, low Wehnelt bias voltages can be used to minimize the gun crossover and limit adverse Boersch effects that increase energy spread. We envision that with the $\mathrm{C} 0$ lens modification, low loss energy resolutionof $0.4 \mathrm{eV}$ can be achieved with currents $>100 \mathrm{nA}$ on the detector, a vast improvement over standard thermionic TEM technology. The C0 lens 
will also allow shorter integration times in stroboscopic pulse mode, minimizing drift and increasing both spatial and energy resolution.

In conclusion, we present the design and realization of a modified TEM for ultrafast dynamic electron microscopy experiments. We showed that despite the radical architectural modification to the machine, high spatial resolution and good energy resolution can be obtained.

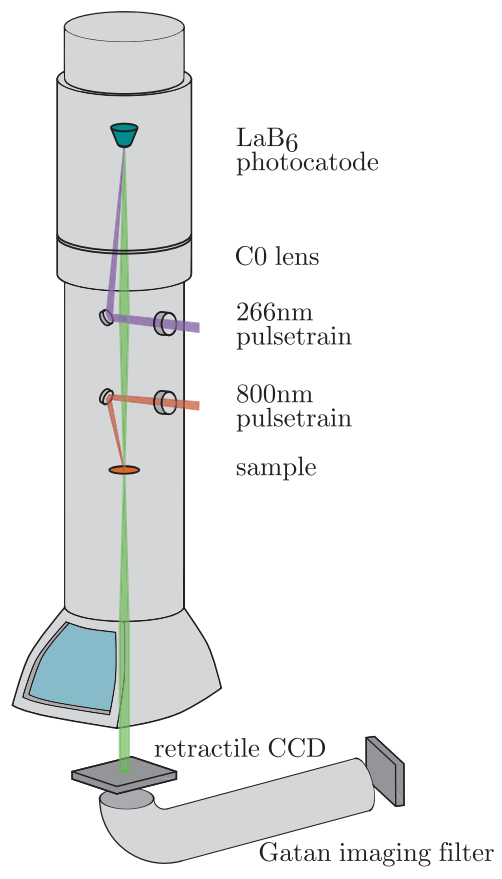

Figure 1: Layout of the system. An optical setup has been designed to allow two laser beams into the column. An ultraviolet beam is directed to the $\mathrm{LaB}_{6}$ tip to produce the probe electron bunches and an infrared pump beam irradiates the sample. An additional lens (C0) has been mounted below the electron gun to collect all the electrons photoemitted.

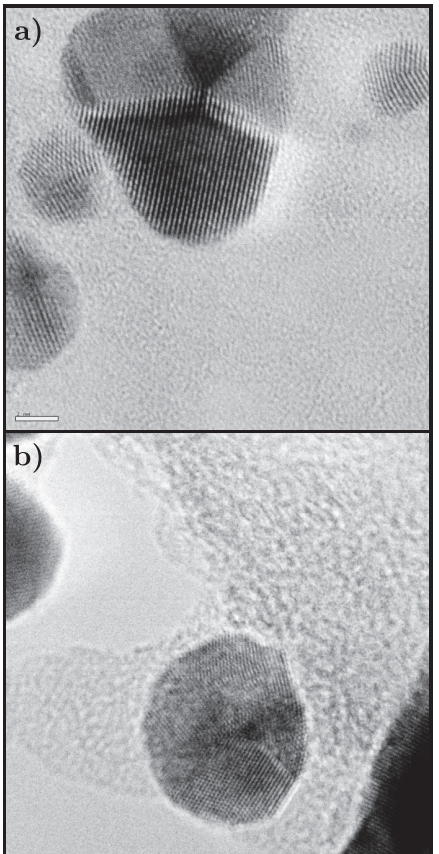

Figure 2: Static TEM images of gold nanoparticles. The image above (a) was acquired before the modifications to the column while the image below (b) was acquired after the $\mathrm{C} 0$ lens and the laser optics had been integrated into the system.
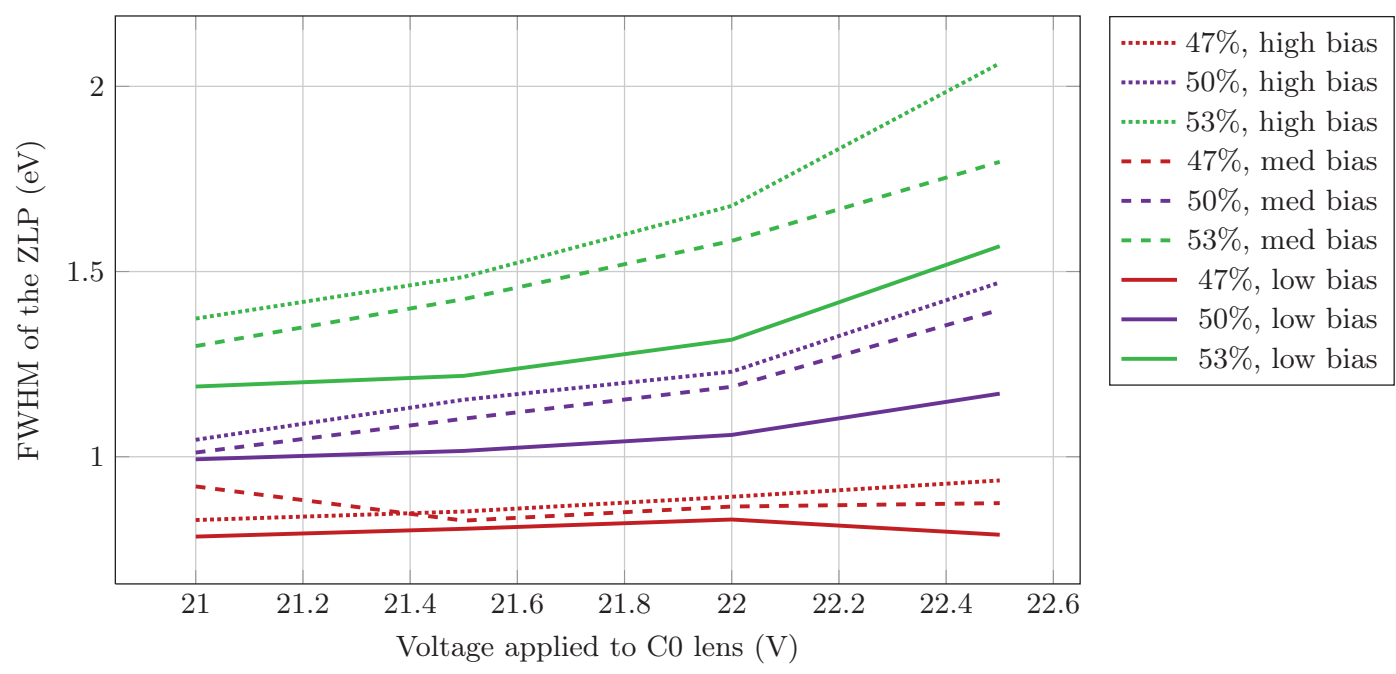

Figure 3: Full width at half maximum of the zero loss peak of EELS spectrum in function of the current in the LaB 6 filament and the bias voltage applied to the tip. 